\title{
Impact of increasing doses of flavonoid-rich and flavonoid-poor fruits and vegetables on vascular reactivity in an 'at risk' group: findings from the FLAVURS study
}

\author{
T. W. George, M. F. Chong, M. H. Gordon, A. M. Minihane, O. B. Kennedy, J. P. E. Spencer \\ and J. A. Lovegrove on behalf of the FLAVURS study group \\ Department of Food and Nutritional Sciences and Institute for Cardiovascular and Metabolic Research (ICMR), \\ University of Reading, Whiteknights, PO Box 226, Reading, Berks RG6 6AP, UK
}

CVD is responsible for $48 \%$ of all deaths each year in Europe ${ }^{(1)}$. Endothelial cell dysfunction is key in early stage development of atherosclerosis $^{(2)}$ leading to CVD. Vascular reactivity and arterial stiffness measured in vivo can be used as markers for general vascular dysfunction. Epidemiological studies indicate that diets rich in fruits and vegetables $(F \& V)$ are associated with reduced risk of CVD ${ }^{(3)}$. However, the relationship between the type and amount of F\&V consumed and CVD risk reduction has not been established. Increasing evidence suggests that dietary flavonoids may explain some of the CVD benefits of increased F\&V intake ${ }^{(4)}$. However, this hypothesis had not previously been tested in an adequately powered, dose-response dietary intervention trial.

FLAVURS (FLAVonoids at University of Reading Study) was a parallel design, randomised, controlled, dietary intervention study which determined the effect of sequentially increasing the dose and type of $F \& V$ consumed in a population predisposed to the development of CVD. Subjects ( $n$ 154, ninety-four females and sixty males) were randomised to either the control (CT), high flavonoid (HF) or low flavonoid (LF) group. The groups were closely matched for gender ratio, age, BMI, smoking status and tea/red wine consumption. After a 2 week run-in period on their habitual diet, subjects were randomised to one of the study groups. The CT group received no dietary manipulation during the 18 week study, while the HF and LF groups sequentially increased their daily F\&V intake (flavonoid-rich or flavonoid-poor, respectively) by an additional two portions every 6 weeks to a maximum of six extra portions. Vascular function was the primary endpoint and was assessed by pulse wave velocity and analysis; digital volume pulse; and laser Doppler imaging with iontophoresis at week 0, 6, 12 and 18. Fasting blood and $24 \mathrm{~h}$ urine samples were also collected at these visits.

There was an increase in arterial stiffness in the CT group over time, that was attenuated by both the HF and LF treatments $(P=0.02)$. There was also a significant time $\times$ treatment interaction on total plasma nitrite (sum of plasma nitrate and nitrite) concentration $(P=0.03)$, a surrogate marker of bioactive nitric oxide (NO). There was an increase in plasma nitrite concentrations in the HF and LF group which reached significance after 12 weeks for the HF group compared with the LF and CT groups $(P=0.02)$. A significant time $\times$ treatment effect on static diastolic blood pressure $(\mathrm{DBP})$ was observed $(P=0.04)$, with a significant decrease in DBP in both the HF and LF group after 6 weeks compared with an increase in the CT group.

The higher total plasma nitrite concentration could reflect an increased endothelial production of NO, stimulated by components in the $\mathrm{F} \& \mathrm{~V}$ such as flavonoids, or the increased endogenous conversion of nitrate found in F\&V to NO. The differences in markers of NO may be responsible for the lower arterial stiffness measured by pulse wave analysis.

This project was funded by the Food Standard Agency (project no. N02039/F5234012).

1. Allender S, Scarborough P \& Peto V (2008) Cardiovascular disease statistics. London: European Heart Network.

2. Bonetti PO, Lerman LO \& Lerman A (2003) Endothelial dysfunction - A marker of atherosclerotic risk. Arterioscler Thromb Vasc Biol 23, 168-175.

3. Hu FB (2003) Plant-based foods and prevention of cardiovascular disease: an overview. Am J Clin Nutr 78, 544S-551S

4. Yeboah J, Crouse JR, Hsu FC et al. (2007) Brachial flow-mediated dilation predicts incident cardiovascular events in older adults. Cardiovasc Health Study 115, 2390-2397. 